\title{
For the greatest benefit to humankind
}

\author{
How has computational science been recognized throughout Nobel history?
}

( n 5 October 2021, the Royal Swedish Academy of Sciences announced that the 2021 Nobel Prize in Physics will be awarded to three scientists for their "groundbreaking contributions to our understanding of complex physical systems". One half of the prize will go to Syukuro Manabe and Klaus Hasselmann for the development of physical models that enabled the simulation of global climate and the prediction of global warming; the other half will be awarded to Giorgio Parisi for the mathematical modeling of complex disordered systems.

The computational science community has certainly been thrilled with this news as it reiterates the essential role of computational science in helping to address the most critical challenges in our society, such as climate change. The mathematical and physical models developed by the three aforementioned researchers formed the foundation to investigate complex systems. The climate model, first proposed by Manabe in the 1960 s by considering the interaction of radiation and vertical mass transportation, successfully predicted the correlation between the carbon dioxide content in the atmosphere and the Earth's surface temperature. Hasselmann's stochastic climate model distinguished reliable climate patterns from chaotic weather, and further differentiated the influence of human activities from that of natural effects on the climate. From a much broader perspective of complex systems, Parisi introduced the idea of 'replica trick' as a new way to mathematically interpret and predict the statistical behavior of disordered systems. This technique is widely used nowadays in various disciplines, including physics, materials science, neuroscience, and machine learning.

This isn't the first time that the contributions of the computational science community have been recognized throughout Nobel history. As a matter of fact, the community has been responsible for many advances in computer code and platforms $s^{1}$ that have indirectly supported many of the awarded scientific discoveries. To name a few, in the 1960s, Martinus Veltman, who shared the 1999 Nobel Prize in Physics with Gerardus 't Hooft, employed the assembly programming language to manipulate algebraic operations that made it possible to solve complex quantum field theory equations ${ }^{2}$, which contributed to better explaining the quantum structure of electroweak interactions in physics. Around the same time, Manabe and colleagues, with the help of Fortran code and digital computers, were able to simulate our climate system. Saul Perlmutter, one of the researchers who shared the 2011 Nobel Prize in Physics, pioneered the use of supercomputers to analyze and validate observational imaging data in cosmology, which contributed to the discovery that the expansion of the Universe is accelerating.

But computational science contributions have not only implicitly helped accelerate scientific discoveries: they have also been explicitly honored with Nobel prizes in the past. Some of these contributions are represented by important mathematical and physical models that not only help us to more accurately understand the laws of nature, but that also allow for the computation of different scientific phenomena that were otherwise hard or impossible to simulate. The climate models developed by Manabe and Hasselmann, for instance, fit this category. Another example is the Marcus theory, proposed by Rudolph A. Marcus, who received the 1992 Nobel Prize in Chemistry. In the mid-1950s, Marcus developed a mathematical model to explain the rate of electron transfer reactions between two chemical species, such as inorganic molecules and biomolecules (for instance, proteins). With many extensions and refinements for specific problems, the main contribution of the Marcus theory was to mathematically model the electron jumping process together with molecular structural change. This theory enabled the accurate computation of redox reactions in important scientific problems — including photosynthesis, enzyme reactions, and corrosion - and was successfully linked with experimental observations. Another notable example is the 1982 Nobel Prize in Physics, which was awarded to Kenneth G. Wilson for his work on phase transitions. Wilson proposed a model based on the renormalization group theory to describe the physics of multi-scale fluctuations, which made numerically computing crucial quantities during phase transition practical at the time. Wilson's finding about the key role of the dimensionality of the order parameter to describe phase transition still inspires new research today that leverages new computing algorithms and the increasing availability of data ${ }^{3}$.

In addition to mathematical models, computational methods have also been explicitly recognized in the past. A clear example of such recognition is the 1998 Nobel Prize in Chemistry, which was shared between Walter Kohn and John Pople. Kohn laid the foundation for the density functional theory in 1954 and demonstrated that the ground state properties of a many-electron system can be described by the ground state electron density in space; in practice, this approximation made quantum chemistry calculations more computationally feasible. Pople is well-known for his pioneering work in developing the first version of Gaussian, and later for his contributions to Q-Chem, which are two of the most widely used computational chemistry tools to date. More recently, in 2013, the Nobel Prize in Chemistry again underscored the importance of computational methods, this time related to the development of the hybrid QM/MM (quantum mechanics/ molecular mechanics) approach for modeling chemical reactions. The ingenious idea treats key elements that are responsible for these reactions with quantum chemistry theory (which allows for more accurate results), while the remainder is described with classical mechanics (which makes the approach more computationally efficient). Among its many important applications to different fields, the QM/MM approach stimulated fruitful research in computational structural biology $y^{4,5}$.

It is worth mentioning that our list of computational science contributions related to Nobel prizes is far from being exhaustive. In addition, for the sake of this Editorial, we focused on the physics and chemistry prizes only. Nevertheless, the contributions highlighted here demonstrate that mathematical and computational methods have become exceptionally relevant in science: in many cases, they have become an essential tool to the most exciting and important scientific discoveries of our society.

What's next? Certainly, this will not be the last time that computational science is recognized by a Nobel prize. Looking ahead, as new computing algorithms and 
architectures are developed and become more sophisticated, such as deep learning and quantum computing, it's not hard to picture computational science in the spotlight again. Be it in physics, chemistry, or medicine, we can surely expect more successful stories and achievements from the computational science community to come.

Published online: 11 November 2021 https://doi.org/10.1038/s43588-021-00169-x
References

1. Perkel, J. M. Nature 589, 344-348 (2021).

2. Day, C. Nobel prizes for computational science. Physics Today (11 October 2013).

3. Yin, J., Pei, Z. \& Gao, M. C. Nat. Comput. Sci. 1, 686-693 (2021).

4. Senn, H. M. \& Thiel, W. Angew. Chem. Int. Ed. 48, 1198-1229 (2009).

5. Levitt, M. Nat. Struct. Mol. Biol. 8, 392-393 (2001). 